\title{
Chronic Coronary Artery Constriction Leads to Moderate Myocyte Loss and Left Ventricular Dysfunction and Failure in Rats
}

\author{
Piero Anversa, Xun Zhang, Peng Li, and Joseph M. Capasso \\ Department of Medicine, New York Medical College, Valhalla, New York 10595
}

\begin{abstract}
Coronary artery narrowing, ranging from $19 \%$ to $61 \%$, was induced in rats and ventricular performance, myocardial damage, and myocyte hypertrophy were examined 1 mo later. Animals were separated into two groups, exhibiting ventricular dysfunction and failure, respectively. Dysfunction consisted of a 2.4fold increase in left ventricular end diastolic pressure (LVEDP), 15\% decrease in left ventricular peak systolic pressure (LVPSP), 24\% reduction in developed pressure (DP), and a 16\% depression in - $\mathrm{dP} / \mathrm{d} t$. Failure was defined on the basis of a 4.7-fold elevation in LVEDP, and a $26 \%, 47 \%, 45 \%$, and $41 \%$ decrease in LVPSP, DP, $+\mathrm{dP} / \mathrm{d} t$, and $-\mathrm{dP} / \mathrm{d} t$. Moreover, in this group, right ventricular end diastolic and systolic pressures increased 5.5- and 1.2-fold. Left and right ventricular weights expanded $23 \%$ and $51 \%$ with dysfunction and $30 \%$ and $56 \%$ with failure. Left ventricular hypertrophy was characterized by ventricular dilation and wall thinning which were more severe in the failing animals. Foci of damage were found in both groups but tissue injury was more prominent in the endomyocardium and in failing rats. Finally, myocyte loss in the ventricle was $10 \%$ and $20 \%$ with dysfunction and failure whereas the corresponding enlargements of the unaffected myocytes were $34 \%$ and $53 \%$. Thus, coronary narrowing led to abnormalities in cardiac dynamics with an increase in diastolic wall stress and extensive ventricular remodeling in spite of a moderate loss of myocytes and compensatory reactive hypertrophy of the viable cells. (J. Clin. Invest. 1992. 89:618-629.) Key words: left and right ventricular dynamics • myocardial damage • myocyte hypertrophy • ventricular remodeling $\bullet$ wall stress
\end{abstract}

\section{Introduction}

In a large number of patients affected by chronic coronary artery disease, the magnitude of constriction of the epicardial coronary arteries by atherosclerosis does not correspond to the severity of the clinical manifestations of myocardial dysfunction and failure (1-7). Similar degrees of coronary stenosis detected angiographically have been found to be associated with variable hemodynamic abnormalities, raising questions on the significance of these fixed obstructions of the coronary tree in the prediction of the short- and long-term outcomes of ischemic heart diseaseclinically (7). This apparent inconsistency

Address reprint requests to Dr. Anversa, Department of Medicine, Vosburgh Pavillion-Room 303, New York Medical College, Valhalla, NY 10595.

Received for publication 10 June 1991 and in revised form $26 \mathrm{Au}$ gust 1991.

J. Clin. Invest.

(c) The American Society for Clinical Investigation, Inc.

$0021-9738 / 92 / 02 / 0618 / 12 \$ 2.00$

Volume 89, February 1992, 618-629 becomes even greater when anatomical findings are taken into account (7-12). Histologic examination of hearts of patients who died of congestive ischemic heart disease reveals that only small quantities of viable myocardium have been replaced by scarred tissue $(9,12)$, suggesting that the overall extent of damage in the ventricular wall may not reflect a loss of myocytes which would be considered critical for the induction of irreversible cardiac failure and death. Studies in humans $(13,14)$ and animals $(15,16)$ have demonstrated that occlusion of a major coronary artery, resulting in acute transmural myocardial infarction, leads to overt cardiac failure when the destruction in muscle mass affects $40-50 \%$ of the myocyte population of the left ventricle. Moreover, ventricular decompensation persists during the healing process (17-19) and long thereafter $(20,21)$. Importantly, a direct relationship exists between healed infarct size and ventricular performance in animals (18) and humans $(22,23)$, further emphasizing potential differences in the genesis of cardiac failure produced by occlusive and nonocclusive coronary stenosis. However, this suspected discrepancy has never been documented and, on the assumption that such a phenomenon is real, there is no knowledge on the mechanisms responsible for the development of congestive heart failure with coronary narrowing. Thus, a relevant question to be addressed is whether partial reductions in luminal diameter of a major epicardial coronary artery result in an impairment of ventricular function when $<40-50 \%$ of myocytes are lost or is this quantity required to generate severe ventricular decompensation, mimicking the condition of myocardial infarctioninduced cardiac failure. In addition, it is still unknown whether comparable losses of myocytes in a segmental fashion, i.e., myocardial infarction, or in a scattered manner across the wall, i.e., ischemic cardiomyopathy, evoke identical hypertrophic reactions on the remaining unaffected cells. Finally, myocytolytic necrosis may persist with time in the presence of a fixed nonocclusive lesion of a major coronary artery and this process may affect ventricular remodeling $(24,25)$ and the recovery of structure and function chronically. Therefore, coronary narrowing was produced in rats and the functional and anatomical variables controlling ventricular wall stress were evaluated 1 mo after surgery. Furthermore, the magnitudes of myocyte loss and reactive cellular hypertrophy were measured morphometrically. The 1-mo interval was chosen because of previous work performed on the effects of total coronary occlusion and myocardial infarction on the determinants of ventricular loading and myocyte growth (21).

\section{Methods}

Experiments were carried out in male Sprague-Dawley rats at 2 mo of age (Charles River Breeding Laboratories, North Wilmington, MA). Coronary artery narrowing was performed in 25 animals. Six animals in this group died within $4 \mathrm{wk}$ after coronary constriction. Nine shamoperated rats served as controls. All animals were killed 1 mo after surgery. 
Coronary artery narrowing. Under ether anesthesia, thoracotomy via the third left intercostal space was performed, the atrial appendage elevated, the left coronary artery located and a suture positioned around the vessel 1-2 mm from its origin. Subsequently, a probe of 275 $\mu \mathrm{m}$ in diameter was held in contact with the wall of the exposed coronary artery. The entire vessel and the probe were ligated and the probe quickly removed $(24,25)$. The chest was closed and the animals were allowed to recover. Sham-operated control rats were treated similarly except that the ligature around the coronary artery was not tied. Since blood supply to the left ventricle in the rat heart is provided by the left descending and septal arteries with no evident circumflex artery (26), coronary constriction near its origin was expected to affect most of the left ventricle.

Functional measurements. Just before sacrifice, animals were anesthetized with chloral hydrate $(300 \mathrm{mg} / \mathrm{kg}$ body weight, ip), and the external right carotid artery was exposed and cannulated with a microtip pressure transducer catheter (model PR 249, Millar) connected to an electrostatic chart recorder (model ES 2000, Gould, Inc. Cleveland, $\mathrm{OH})$. After monitoring arterial blood pressure, the catheter was advanced into the left ventricle for the evaluation of left ventricular pressures and $\mathrm{d} P / \mathrm{d} t$. Subsequently, a second catheter (Millar PR 249) with a $120^{\circ}$ curved tip was inserted in the right jugular vein and advanced through the superior vena cava and the right atrium into the right ventricular chamber for the measurements of right ventricular pressures and $\mathrm{d} P / \mathrm{d} t$. Thus, measurements were made of systolic, diastolic, and mean arterial blood pressures and ventricular pressures and $\mathrm{d} P / \mathrm{d} t$ in the closed chest preparation.

Fixation procedure. At the completion of the hemodynamic determinations, all animals were killed by arresting the heart in diastole with $1 \mathrm{ml}$ of cadmium chloride ( $100 \mathrm{mM}$, i.v.). The heart was then perfused retrogradely through the abdominal aorta as previously described (15). Perfusion pressure was adjusted to the mean arterial pressure measured in vivo. The left ventricular chamber was filled with fixative and kept at a pressure equal to the in vivo measured end-diastolic pressure throughout the fixation procedure (24). After perfusion with $\mathrm{pH} 7.2$ phosphate buffer for $3 \mathrm{~min}$, the coronary vasculature was perfused for 15 min with a solution containing $2 \%$ paraformaldehyde and $2.5 \%$ glutaraldehyde (15). Subsequently, the heart was excised and the weights of the left ventricle including the septum and the right ventricle were recorded. It should be noted that in 2 of the 19 coronary constricted rats perfusion fixation was not adequate. Therefore, quantitative morphology was restricted to 17 experimental rats.

Degree of coronary artery narrowing. The initial 2-3-mm segment of the left coronary artery was dissected free to localize the level of coronary constriction. The vessel was then cut transversely to expose the lumen of the coronary artery at the region of the ligature. The luminal diameter of the vessel adjacent to the narrowed site and in the constricted portion were measured with a dissecting microscope having an incorporated ocular reticle. Maximal and minimal internal diameters in each of these two locations were determined at $\times 20$, and the geometric mean value was calculated $(24,25)$. The degree of constriction was evaluated by comparing the vessel diameter above the stenosis with the diameter at the level of the stenosis. This approach was preferred to comparisons between experimental and sham-operated control rats, in order to minimize the variability in the coronary vasculature among animals.

Ventricular dimension. The major intracavitary axis of the left ventricle from apex to base was measured by sectioning the elliptical chamber parallel to the longitudinal diameter. Subsequently, each left ventricle was serial-sectioned into $\sim 2$-mm-thick rings perpendicular to the longitudinal axis to obtain six equally spaced parallel planes from the base to the apex in which the average wall thickness of the free wall and chamber luminal diameter were measured. Eight measurements of the left ventricular free wall in each section were collected and their values averaged. The minimal and maximal diameters of the ventricular chamber at each of the six sampled levels were determined and their geometric mean was computed (27). The long and transverse diameters were employed to calculate chamber volume (27). Right ventricular wall thickness near the base, in the portion containing the coronary artery, was also measured by averaging 10 determinations in each heart. Moreover, the measurements of wall thickness, chamber radius, and ventricular end diastolic pressure in the left ventricle were used to compute diastolic transmural wall stress (28) at each of the six sites examined from base to apex. Systolic transmural wall stress was also calculated in a similar manner $(24,28)$.

Tissue sampling. In all 27 animals, the two middle slices of the left ventricle, nearly halfway between the apex and the base, were cut in thinner sections in order to obtain three to four slices, $\sim 1 \mathrm{~mm}$ in thickness, which were subsequently postfixed in osmium, dehydrated in acetone, and flat embedded in araldite. Sections 1-2 $\mu \mathrm{m}$ thick were cut with a glass knife, $38 \mathrm{~mm}$ long (model 2078 Histoknife Maker, LKB Instruments, Gaithersburg, MD), and a rotary microtome (HM 350, Microm, Carl Zeiss, Inc., Thornwood, NY). These sections, which contained the entire thickness of the wall, were stained with toluidine blue for quantitative measurements. 15 consecutive fields, each from the endomyocardium and epimyocardium in each animal, were examined at a calibrated magnification of $\times 400$ with a reticle containing 42 sampling points (105844, Wild Heerbrugg Instruments, Inc., Farmingdale, NY). This reticle defined an uncompressed tissue area of 62,500 $\mu \mathrm{m}^{2}$, which was employed to determine the number of lesions represented by foci of damage per unit area of myocardium. Moreover, the fraction of points lying over these foci was measured to compute the volume fraction of lesions in the myocardium and the average crosssectional area of the lesion profiles. Sarcomere length was measured in the midregion of the wall at $\times 1,000$ by averaging groups of 10 sarcomeres each. 10 such determinations were collected in each left ventricle.

Sites in these slices with myofibers oriented in the longitudinal or transverse direction were cut free with a razor blade from the thick embedded sections. Approximately 25-30 blocks collected from each animal were then reembedded in small flat molds (29).

Myocyte size and number. Ten randomly chosen tissue blocks from each left ventricle were sectioned at a thickness of $0.75 \mu \mathrm{m}$ and stained with toluidine blue. Morphometric sampling at a magnification of $\times 1,000$ consisted of counting the number of myocyte nuclear profiles, $N(n)$, in a measured area, $A$, of tissue sections in which cardiac muscle fibers were cut transversely. A square tissue area of $9,950 \mu \mathrm{m}^{2}$ was delineated in the microscopic field by the 42 sampling points ocular reticle. A total of 20-26 such fields were evaluated in the endomyocardial and epimyocardial regions of each ventricle of each animal to determine the number of nuclear profiles per unit area of myocardium, $N(n)_{A}$, and the volume fraction of myocytes, $V(m)_{V}$, in the myocardium of the inner and outer layers of the wall.

Nuclear length, $D(n)$, was determined in the endomyocardial and epimyocardial regions of each left ventricle from 50 measurements each made at $\times 1,250$ in longitudinally oriented myocytes viewed with a microscope having an ocular micrometer accurate to $0.5 \mu \mathrm{m}$. Ten blocks with myofibers sectioned parallel to their length were cut, sections at $\sim 2 \mu \mathrm{m}$ in thickness collected and stained, and 10 measurements of nuclear length were recorded from each tissue section, 5 each for each region of the wall.

From the estimation of $N(n)_{A}$ and $D(n)$, the number of myocyte nuclei per unit volume of myocardium, $N(n)_{V}$, was computed using the equation $(15,29)$ :

$N(n)_{V}=N(n)_{A} / N(n)_{V}$

Myocyte cell volume per nucleus in the inner and outer layers of the wall of each left ventricle, $V(m)_{n}$, was obtained from the volume fraction of myocytes, $V(m)_{V}$, divided by the number of myocyte nuclei per unit volume of myocardium:

$V(m)_{n}=V(m)_{V} / N(n)_{V}$

The total number of myocyte nuclei in each ventricle, $N(n)_{T}$, was then derived from the product of the number per unit volume, $N(n)_{V}$, 
and the total ventricular volume of viable myocardium, $V_{T}$, previously measured from the large sections of the ventricle.

$N(n)_{T}=N(n)_{V} \times V_{T}$

In this case, $N(n)_{V}$ was evaluated by averaging the values obtained in the inner and outer layers of the wall in each ventricle. A similar approach was used when regional morphometric values of volume fraction of tissue components, numerical density of nuclei, nuclear length, and cell volume per nucleus were expressed as indicators of the entire wall. The theoretical aspects and practical applications of the morphometric procedure briefly summarized above have recently been described in detail (30).

Sampling size. The magnitude of sampling utilized in this investigation was selected on the basis of previous work performed in our laboratory and the principle of Poisson statistics (30). The latter can be used as a reasonable guideline for morphometric data collection since it provides a more conservative estimate of necessary counts than more specific formulations derived for point counts and profile counts (20). By assuming that biological variability among animals in a given experimental group is $\sim 10 \%$, counting errors in each animal should also be limited by the same order of magnitude for the least frequent structure (20). In the present study, the area of myocardium sampled yield an average of 168,173 , and 341 nuclear profile counts for the endomyocardium, epimyocardium and wall of each coronary constricted left ventricle, respectively. Corresponding sampling errors for these values were $7.7 \%, 7.6 \%$, and $5.4 \%$. Counts in control animals were 172, 180, and 352 which yielded sampling errors per animal of $7.6 \%, 7.4 \%$, and $5.3 \%$.

Data collection and analysis. All tissue samples were coded and the code was broken at the end of the experiment. Results are presented as mean \pm SD computed from the average measurements obtained from each rat. Statistical significance for comparison between two measurements within the wall of each ventricle was determined using the paired Student's $t$ test. Statistical significance in multiple comparisons among independent groups of data, in which analysis of variance and the $F$ test indicated the presence of significant differences, was determined by the Scheffe method (31). Values of $P<0.05$ were considered to be signifcant. Because measurements presented were not obtained in all animals, $n$ values for each parameter measured are listed in the text or the legend of each figure.

\section{Results}

The surgical procedure employed for the imposition of a nonocclusive stenosis of the left coronary artery near its origin resulted in reductions in luminal diameter ranging from $19 \%$ to $61 \%$. Physiological measurements of cardiac dynamics demonstrated that coronary artery narrowing affected heart function in a variable fashion and this impairment was not correlated with the degree of constriction. This phenomenon has previously been described $45 \min (24)$ and $5 \mathrm{~d}(25)$ after coronary stenosis. Because of the differences in ventricular performance, the 19 animals with vessel narrowing were subdivided into two groups of 8 and 11 rats, respectively. The first group included animals in which left ventricular developed pressure was $\geq 75$ $\mathrm{mm} \mathrm{Hg},+\mathrm{d} P / \mathrm{d} t$ was $\geq 9,000 \mathrm{~mm} \mathrm{Hg} / \mathrm{s}$ and $-\mathrm{d} P / \mathrm{d} t$ was $\geq 8,000 \mathrm{~mm} \mathrm{Hg} / \mathrm{s}$. Moreover, no alterations in right ventricular performance were noted. The second group included rats with values of left ventricular developed pressure, $+\mathrm{d} P / \mathrm{d} t$ and $-\mathrm{d} P / \mathrm{d} t$ below those stated above. At times, abnormalities in right ventricular function were also present. The degree of coronary constriction in the first and second group of rats was $46 \pm 12 \%(n=8)$ and $43 \pm 18 \%(n=11)$, respectively. These changes in diameter corresponded to a $69 \pm 15 \%$ and $64 \pm 19 \%$ reduction in luminal cross-sectional area. On the basis of the
Table I. Effects of Coronary Artery Constriction on Arterial Blood Pressure and Heart Rate

\begin{tabular}{lccc}
\hline & & \multicolumn{2}{c}{ Narrowed animals } \\
\cline { 3 - 5 } & $\begin{array}{c}\text { Sham-operated } \\
\text { animals } \\
(n=9)\end{array}$ & $\begin{array}{c}\text { Group 1 } \\
(n=8)\end{array}$ & $\begin{array}{c}\text { Group 2 } \\
(n=11)\end{array}$ \\
\hline Body weight (g) & $381 \pm 22$ & $395 \pm 18$ & $410 \pm 21^{*}$ \\
Arterial blood pressure $(\mathrm{mm} \mathrm{Hg})$ & & & \\
Systolic & $119 \pm 7$ & $100 \pm 7^{*}$ & $87 \pm 12^{* \neq}$ \\
Diastolic & $86 \pm 10$ & $66 \pm 8^{*}$ & $65 \pm 10^{*}$ \\
Mean & $97 \pm 8$ & $77 \pm 7^{*}$ & $72 \pm 11^{*}$ \\
Heart rate $(b p m)$ & $355 \pm 47$ & $314 \pm 43$ & $351 \pm 51$
\end{tabular}

Results are shown as mean $\pm \mathrm{SD}$. * Value that is statistically significantly different from the corresponding value in control animals, $P$ $<0.05$. $^{\ddagger}$ Value that is statistically significantly different from the corresponding value in group 1 animals, $P<0.05$.

hemodynamic profile (see below), it was concluded that these rats exhibited left ventricular dysfunction (group 1) and left ventricular failure (group 2).

Global cardiac performance. Table I shows that body weight was similar in controls and in the first group of rats with coronary constriction, whereas an $8 \%$ increase was measured in the second group of experimental animals. Moreover, systolic, diastolic, and mean arterial pressures were decreased $16 \%, 23 \%$, and $21 \%$ in the first group and $27 \%, 24 \%$, and $26 \%$ in the second group of rats with coronary stenosis. However, heart rate was not different in control and coronary narrowed rats.

Fig. 1 illustrates that alterations in left ventricular minimal diastolic pressure (LVMDP), ${ }^{1}$ end-diastolic pressure (LVEDP), peak systolic pressure (LVPSP), and developed pressure (DP) occurred as a result of coronary stenosis. In the first group of rats LVEDP increased 2.4-fold $(P<0.02)$, and LVPSP and DP decreased $15 \%(P<0.002)$ and $24 \%(P<0.0001)$. However, in the second group, LVMDP and LVEDP augmented 9.7-fold $(P$ $<0.001)$ and 4.7 -fold $(P<0.0001)$, whereas LVPSP and DP diminished 26\% $(P<0.0001)$ and 47\% $(P<0.0001)$. Moreover, in comparison with the first group of coronary stenosed rats, animals in the second group showed values of LVMDP and LVEDP which were 5.7-fold $(P<0.001)$ and 1.9-fold $(P$ $<0.001)$ greater, and values of LVPSP and DP which were $13 \%$ $(P<0.03)$ and $31 \%(P<0.0001)$ lower.

Fig. 2 demonstrates that in the first group of coronary artery narrowed rats $+\mathrm{d} P / \mathrm{d} t$ remained essentially constant while $-\mathrm{d} P / \mathrm{d} t$ decreased $16 \%(P<0.03)$. However, the reduction in $-\mathrm{d} P / \mathrm{d} t$ was not sufficient to affect the $+\mathrm{d} P / \mathrm{d} t$ to $-\mathrm{d} P / \mathrm{d} t$ ratio. In the second group of stenosed rats $+\mathrm{d} P / \mathrm{d} t$ and $-\mathrm{d} P / \mathrm{d} t$ were reduced by $45 \%(P<0.0001)$ and $41 \%(P<0.0001)$ and these changes maintained the $+\mathrm{d} P / \mathrm{d} t$ to $-\mathrm{d} P / \mathrm{d} t$ ratio substantially constant. On the other hand, when the two experimental animal groups were compared, the second group had values of $+\mathrm{d} P / \mathrm{d} t$ and $-\mathrm{d} P / \mathrm{d} t$ which were $38 \%(P<0.0001)$ and $29 \%(P$ $<0.001)$ lower than those in the first group. Moreover, an $11 \%$

1. Abbreviations used in this paper: DP, developed pressure; LVEDP, left ventricular end-diastolic pressure; LVMDP, left ventricular minimal diastolic pressure; LVPSP, left ventricular peak systolic pressure. 

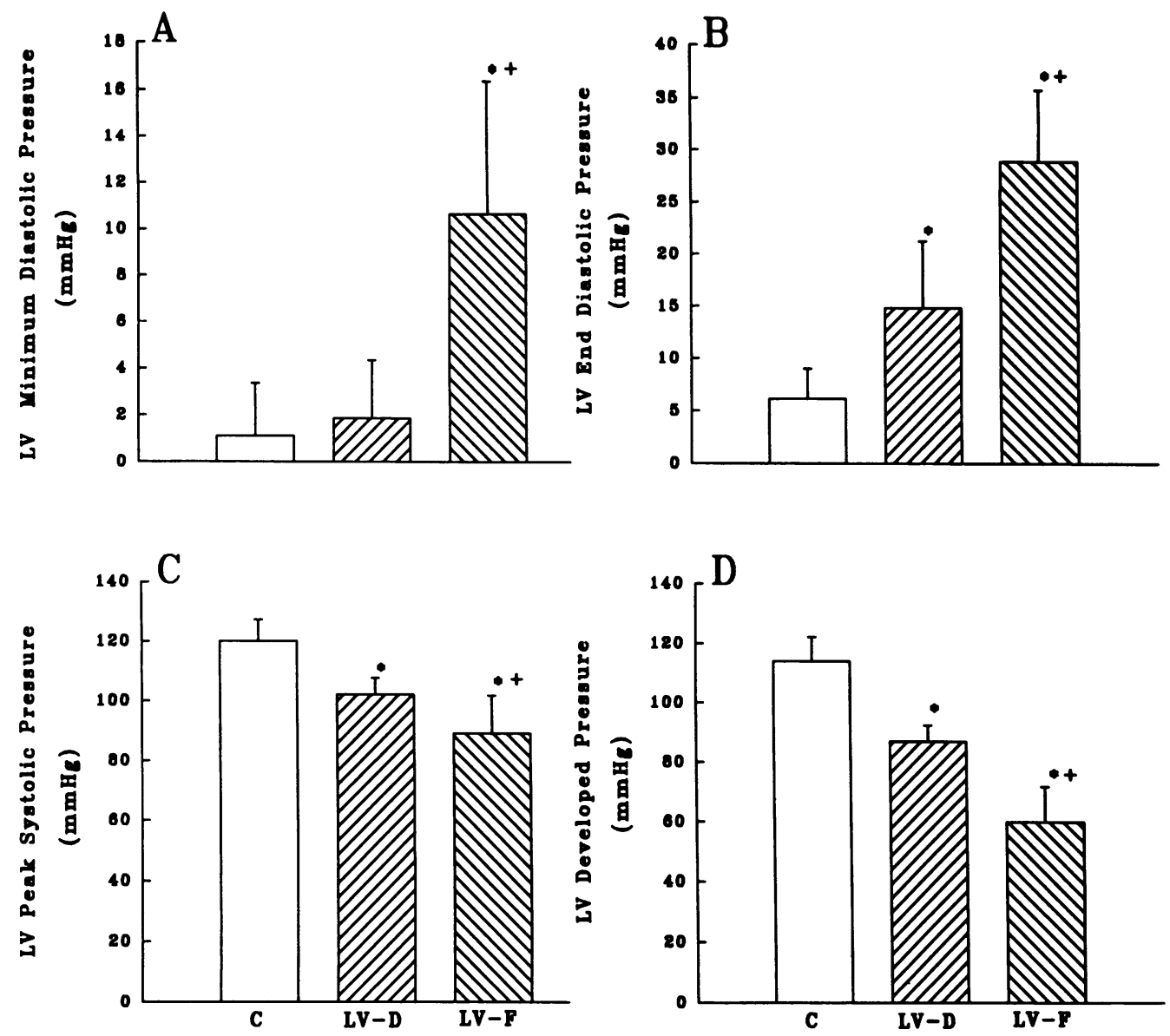

Figure 1. Effects of chronic coronary artery narrowing on left ventricular pressures in animals with left ventricular dysfunction $(L V-D)$ and left ventricular failure $(L V$ $F)$. Results are presented as mean \pm SD. *Value significantly different from the corresponding result in control animals $(C) .{ }^{+}$Value significantly different from the corresponding result in animals with left ventricular dysfunction. (C, $n=9$; LV-D, $n=8 ; \mathrm{LV}-\mathrm{F}, n=11$.)

$(P<0.02)$ reduction in $+\mathrm{d} P / \mathrm{d} t$ to $-\mathrm{d} P / \mathrm{d} t$ ratio was also noted. Although right ventricular function was preserved in the first group of rats, in the second group of animals right ventricular end-diastolic pressure (control, 1.12 \pm 1.19 ; group 1, 1.99 \pm 1.15 ; group $2,6.16 \pm 2.21 \mathrm{~mm} \mathrm{Hg}$ ) and peak systolic pressure (control, 27.57 \pm 1.89 ; group 1, 29.76 \pm 2.39 ; group 2, 32.81 \pm 3.20 $\mathrm{mm} \mathrm{Hg})$ increased 5.5-fold $(P<0.0001)$ and 1.2-fold $(P$ $<0.001$ ), respectively.

In summary, chronic coronary artery constriction resulted in an impairment of myocardial performance ranging from left ventricular dysfunction (group 1) to left ventricular failure (group 2).

Gross cardiac characteristics. Coronary constriction resulted in an increase in weight of the left (control, 805 \pm 83 ; group 1, 987 \pm 94 ; group 2, 1,050 $\pm 90 \mathrm{mg}$ ) and right (control, $234 \pm 16$; group 1,354 \pm 57 ; group 2, $364 \pm 55 \mathrm{~mm} \mathrm{Hg}$ ) ventricles and these augmentations in myocardial mass were similar in animals with left ventricular dysfunction and failure. Left and right ventricular weights expanded 23\% $(P<0.002)$ and $51 \%(P$ $<0.0001)$ in group 1 rats, and $30 \%(P<0.001)$ and $56 \%(P$ $<0.0001)$ in group 2 rats. These changes provoked a $29 \%(P$ $<0.0001)$ and $36 \%(P<0.0001)$ greater heart weight in animals with cardiac dysfunction and failure, respectively (control, 1,039 \pm 100 ; group 1, 1,341 \pm 102 ; group 2, 1,414 $\pm 101 \mathrm{~mm}$ $\mathrm{Hg}$ ). Because of the small variations in body weight among the experimental and control animal groups (Table I), the increases in heart weight and left and right ventricular weight-tobody weight ratios after coronary stenosis were similar to those found with respect to weight changes alone (Data not shown).
In summary, chronic coronary artery narrowing led to biventricular hypertrophy. Myocardial hypertrophy, however, was significantly greater in the right than in the left ventricle.

Ventricular size and shape. Figs. 3 and 4 illustrate the changes in wall thickness (Fig. 3) and chamber diameter (Fig. 4) after coronary artery constriction at each of the six levels of the left ventricle from base to apex. In comparison with controls, group 1 and group 2 rats showed similar alterations in these anatomical parameters. Wall thickness in group 1 decreased, respectively $19 \%(P<0.01), 19 \%(P<0.01), 16 \%(\mathrm{NS})$, $11 \%(\mathrm{NS}), 14 \%$ (NS), and $32 \%(P<0.001)$, at levels $1,2,3,4,5$, and 6. Corresponding changes in group 2 were $-18 \%(P$ $<0.01),-18 \%(P<0.01),-17 \%$ (NS), $-16 \%$ (NS), $-20 \%(P$ $<0.01)$, and $-35 \%(P<0.0001)$. Average right ventricular wall thickness was increased $46 \%(P<0.004)$ and $30 \%(P<0.04)$, in group 1 and group 2 animals (controls, $0.80 \pm 0.10 \mathrm{~mm}$; group $1,1.17 \pm 0.22 \mathrm{~mm}$; group $2,1.04 \pm 0.23 \mathrm{~mm}$ ).

In contrast to the thinning of the wall, ventricular chamber diameter (Fig. 4) increased as a result of coronary artery constriction. In animals with ventricular dysfunction, cavitary diameter expanded $17 \%$ (NS), 24\% ( $P<0.004), 21 \%(P<0.01)$, $13 \%$ (NS), $3 \%$ (NS), and $-18 \%$ (NS) from base to apex. In the presence of ventricular failure, the augmentations in chamber diameter were $22 \%(P<0.01), 32 \%(P<0.0001), 32 \%(P$ $<0.0002), 23 \%(P<0.002), 11 \%(\mathrm{NS})$, and $-2 \%$ (NS). Moreover, the longitudinal axis of the ventricular cavity was $11.95 \pm 0.79 \mathrm{~mm}$ in controls, $15.56 \pm 0.39 \mathrm{~mm}$ in group 1 , and $15.86 \pm 0.48 \mathrm{~mm}$ in group 2 . Thus, this anatomical parameter increased $30 \%(P<0.0001)$ and $33 \%(P<0.0001)$ in animals 

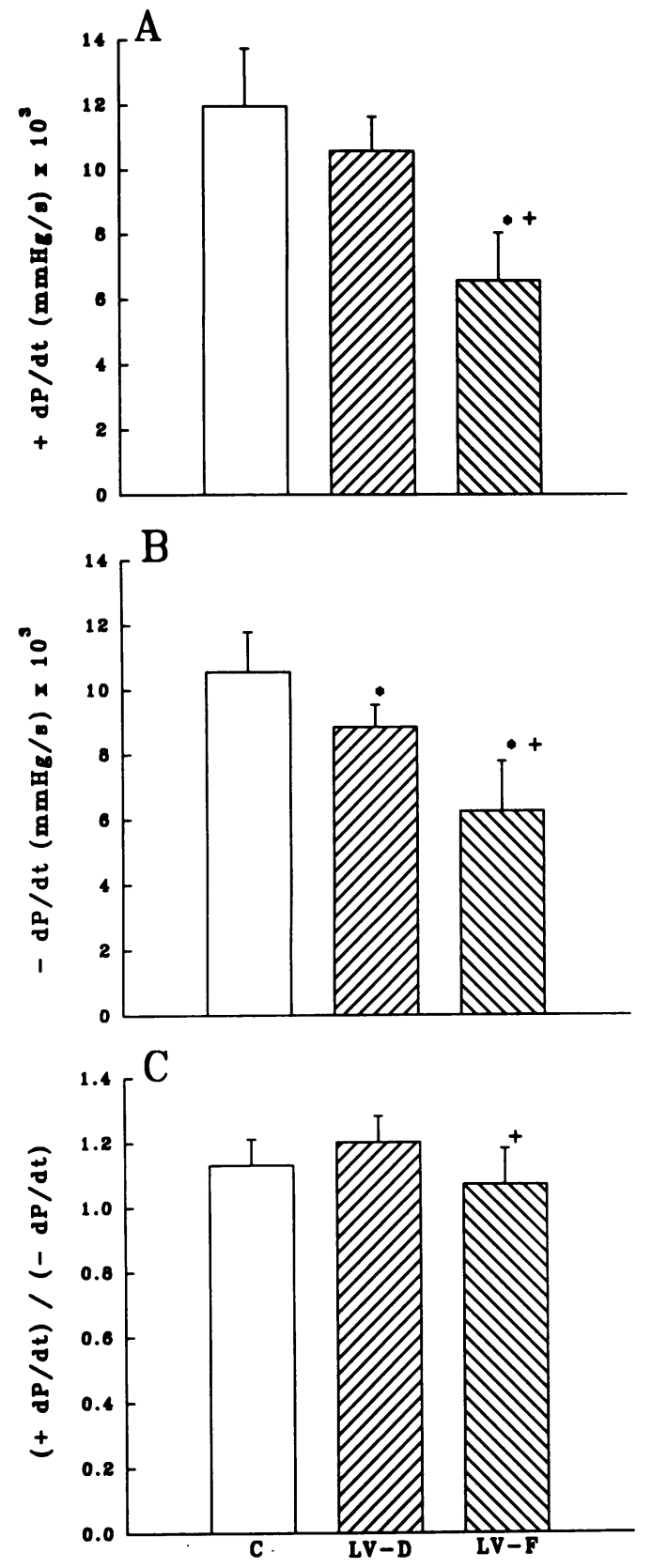

Figure 2. Effects of chronic coronary artery narrowing on left ventricular rate of pressure rise $(+\mathrm{d} P / \mathrm{d} t)$ and decay $(-\mathrm{d} P / \mathrm{d} t)$, and their ratio. Results are presented as mean $\pm \mathrm{SD}$. *Value significantly different from the corresponding result in control animals $(C){ }^{+}$Value significantly different from the corresponding result in animals with left ventricular dysfunction. (C, $n=9$; LV-D, $n=8$; LV-F, $n=11$.)

with ventricular dysfunction and failure, respectively. These changes resulted in marked increases in cavitary volume, $63 \%$ $(P<0.004)$ in group 1 and 93\% $(P<0.0001)$ in group 2 (con-

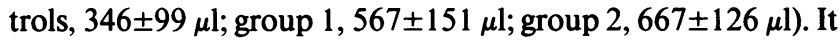
should be pointed out that the shape of the heart was also affected by coronary narrowing, since the ratios of transverse cavitary diameters and longitudinal axis, decreased $13 \%(P$ $<0.03), 22 \%(P<0.01)$, and $37 \%(P<0.001)$ in the last three levels towards the apex in animals with dysfunction, and $18 \%$ $(P<0.01)$ and $26 \%(P<0.005)$ in the two apical rings with failure (data not shown). In addition, the ratios of wall thick- ness-to-chamber radius decreased from base to apex in both groups of experimental animals. Finally, average sarcomere length was $2.01 \pm 0.03 \mu \mathrm{m}$ in controls, $2.06 \pm 0.04 \mu \mathrm{m}$ in animals with left ventricular dysfunction, and $2.15 \pm 0.04 \mu \mathrm{m}$ in animals with left ventricular failure.

In summary, chronic coronary artery constriction led to an increase in ventricular chamber volume, a decrease in left ventricular wall thickness and a change in shape of the heart towards a more elliptical configuration.

Wall stress. The measurements of wall thickness, chamber diameter, and ventricular pressures have been employed to compute the distribution of systolic and diastolic wall stress from the endocardium to the epicardium in each of the six layers examined (Fig. 5). Whereas diastolic wall stress increased significantly throughout the wall and from base to apex after coronary constriction, an opposite phenomenon was observed in terms of systolic wall stress. The greater depressions in ventricular pump performance were associated with the larger increases in diastolic wall stress whereas systolic wall stress decreased.

In summary, chronic coronary artery narrowing produced an elevation in diastolic wall stress and a decrease in systolic wall stress which were proportional to the degree of impairment in ventricular dynamics.

Myocardial damage. Consistent with previous observations $(24,25)$, light microscopic examination of the left ventricular myocardium showed that multiple foci of acute and chronic tissue and myocyte injury were present in coronary stenosed rats. Acute lesions consisted of isolated myocytes with focal or extensive loss of cytoplasmic structures and appearance of large cytoplasmic vacuoles. Chronic damage was characterized by focal loss of myocytes and consequent reparative processes in different phases of healing. The morphometric analysis of myocardial injury associated with coronary constriction did not distinguish among these different forms of tissue and myocyte damage. They were considered part of the same process, simply in various stages of evolution, and consequently combined in this quantitative evaluation.

Fig. 6 demonstrates that the volume percent of damage in the myocardium increased markedly as a result of coronary narrowing. In comparison with sham-operated controls, this structural parameter increased 13 -fold $(P<0.005), 12$-fold $(P$ $<0.001)$, and 12 -fold $(P<0.001)$ in the endomyocardium, epimyocardium, and across the wall of animals with left ventricular dysfunction. In the presence of left ventricular failure, these increases were 22 -fold $(P<0.0001)$, 19-fold $(P<0.0001)$, and 20 -fold $(P<0.0001)$, respectively. Moreover, animals in this group had values of volume fraction of tissue injury which were $72 \%(P<0.02), 57 \%(P<0.03)$, and 64\% $(P<0.002)$ higher than those measured in the inner and outer layers of the ventricle and in the wall of rats with ventricular dysfunction only.

Fig. 7 shows that the size of the lesion profiles in the myocardium was approximately two- to threefold greater in animals with coronary constriction than in controls. Although these sites were consistently larger in animals with ventricular failure than in those with myocardial dysfunction, only the $49 \%$ difference found in the endomyocardium was statistically significant $(P<0.01)$. Moreover, the number of lesion profiles per square millimeter of tissue increased 6.9-fold $(P<0.001)$, 5.8-fold $(P<0.0001)$, and 6.3-fold $(P<0.0001)$, in the endomyocardium, epimyocardium, and as an average across the 

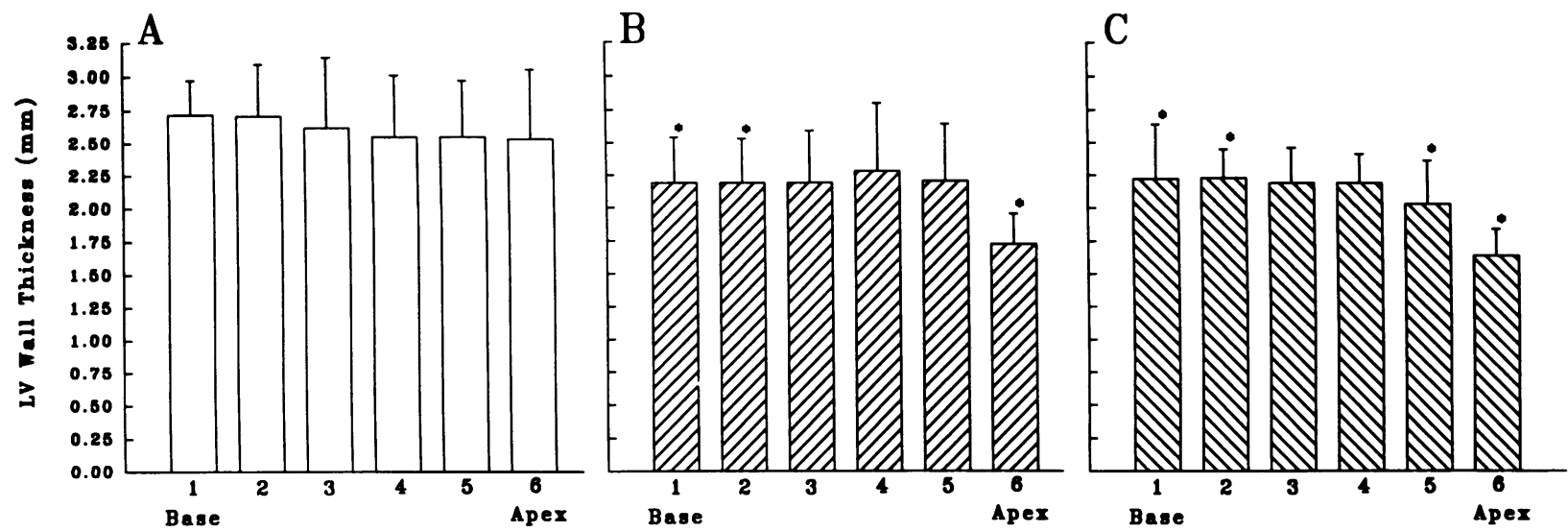

Figure 3. Effects of chronic coronary artery narrowing on left ventricular wall thickness from the basal to the apical region. $(A)$ Controls; $(B)$ left ventricular dysfunction; $(C)$ left ventricular failure. Results are presented as mean $\pm \mathrm{SD}$. *Value significantly different from the corresponding result in control animals $(C)$. ${ }^{+}$Value significantly different from the corresponding result in animals with left ventricular dysfunction. (C, $n=9$; LV-D, $n=8 ;$ LV-F, $n=11$.)

wall in rat with ventricular dysfunction (Fig. 8). Corresponding increases in ventricular failure were 7.9-fold $(P<0.0001), 7.7$ fold $(P<0.0001)$, and 7.8 -fold $(P<0.0001)$.

In summary, the magnitude of chronic myocardial damage induced by coronary artery constriction was greater in the presence of ventricular failure than myocardial dysfunction.

Number of myocyte nuclei in the ventricle. The primary measurements that were used for the derivation of the numerical density of myocyte nuclei per unit volume of myocardium consisted in the determination of the number of myocyte nuclei per unit area of nondamaged tissue and in the evaluation of average nuclear length. These parameters were obtained in the endomyocardium and epimyocardium of each ventricle and then combined to compute an average value in the wall (data not shown). The product of these average values combined with the aggregate volume of viable myocardium in the ventricles yielded the total number of myocyte nuclei in the ventricles $(15,16,21,32)$. The results obtained in control and coronary constricted rats are shown in Fig. 9.

In comparison with sham-operated control animals, coronary narrowed rats showing ventricular dysfunction exhibited a $10 \%(P<0.04)$ loss in the total number of myocyte nuclei of the left ventricular wall. A greater loss in myocyte nuclei was found with ventricular failure (Fig. 9). With respect to control value, a $20 \%(P<0.0001)$ reduction in nuclei was demonstrated in this group. Moreover, this parameter was $12 \%(P$ $<0.02)$ lower in animals with cardiac failure than in those with myocardial dysfunction. It should be noted that these decreases in the total number of myocyte nuclei in the ventricle correspond to identical changes in myocyte number if the proportion between mononucleated and binucleated cells in the myocardium remains essentially constant (32).

In summary, chronic coronary artery constriction led to myocyte loss which was greater with ventricular failure than with myocardial dysfunction.

Myocyte cell volume. Fig. 10 shows the measurements of myocyte cell volume per nucleus obtained in the left ventricle of control and coronary narrowed rats. This cellular parameter was not statistically different in the endocardial and epicardial layers of the wall in sham-operated controls, and the regional increases with ventricular dysfunction did not affect this characteristic. Myocyte cell volume per nucleus in this group in-
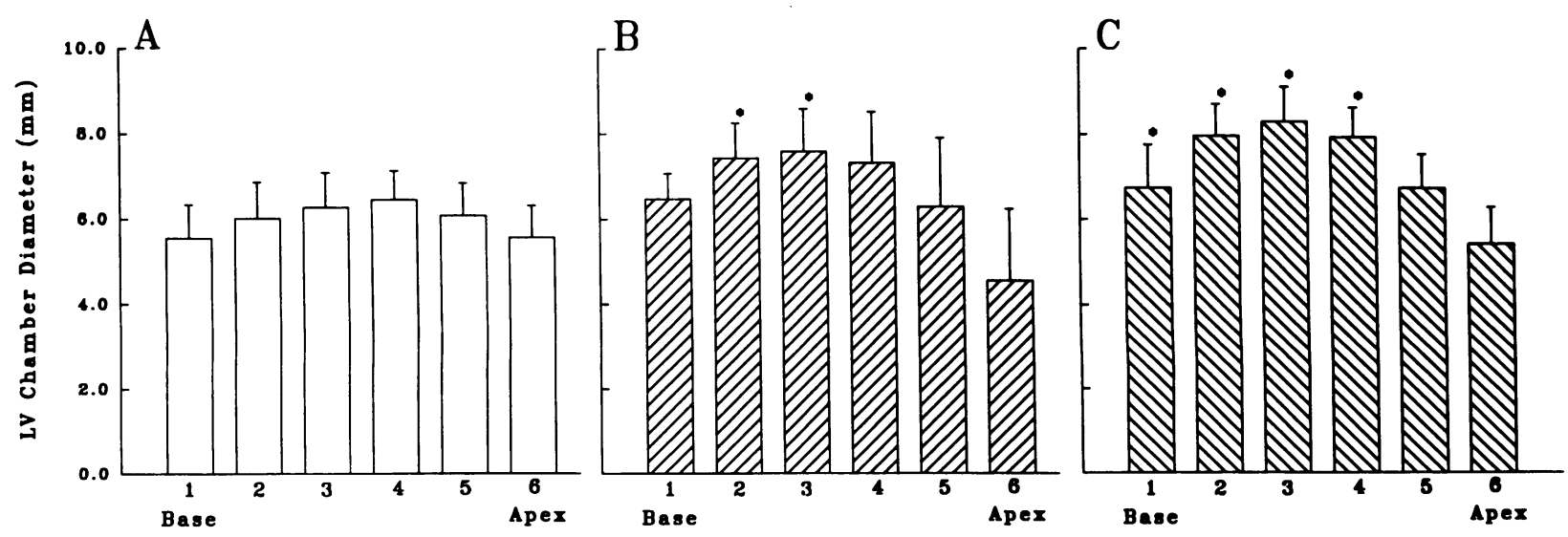

Figure 4. Effects of chronic coronary artery narrowing on left ventricular chamber diameter from the basal to the apical region. $(A)$ Controls; $(B)$ left ventricular dysfunction; $(C)$ left ventricular failure. Results are presented as mean $\pm \mathrm{SD}$. *Value significantly different from the corresponding result in control animals $(C)$. ${ }^{+}$Value significantly different from the corresponding result in animals with left ventricular dysfunction. (C, $n$ $=9 ; \mathrm{LV}-\mathrm{D}, n=8 ; \mathrm{LV}-\mathrm{F}, n=11$.) 

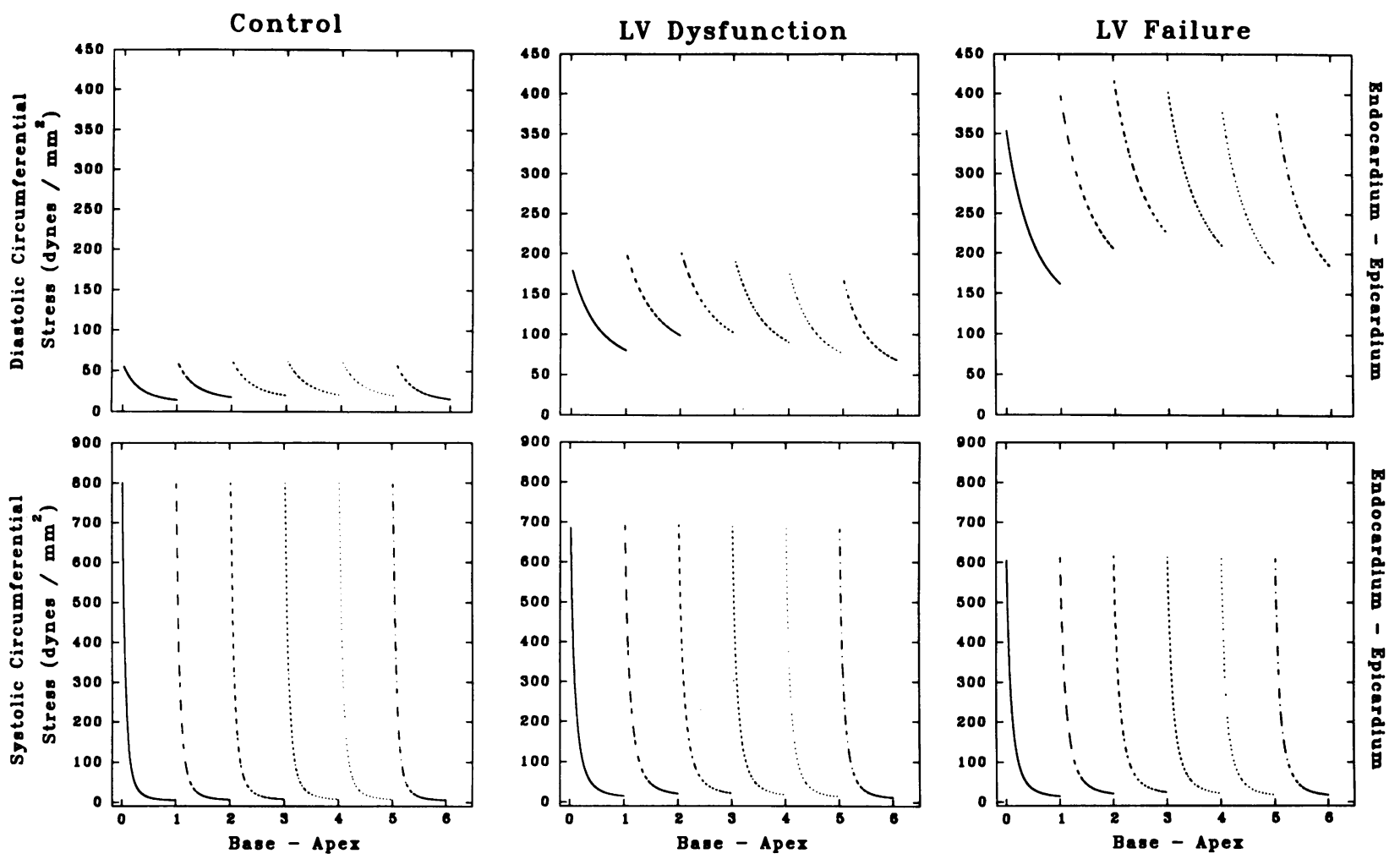

Figure 5. Effects of chronic coronary artery narrowing on left ventricular diastolic and systolic wall stress, from the basal to the apical region of the ventricle and from the endocardium to the epicardium. The increases in diastolic wall stress with dysfunction and failure are all significant and the values in the failing group are all higher than those in the dysfunction group. The decreases in systolic wall stress with narrowing are significant only in the inner one third of the wall of both experimental animal groups and the reduction with failure is greater than with dysfunction. (C, $n=9$; LV-D, $n=8 ; \mathrm{LV}-\mathrm{F}, n=11$.)

creased 39\% $(P<0.0001)$ in the endomyocardium and $29 \%(P$ $<0.0001)$ in the epimyocardium resulting in an average $34 \%(P$ $<0.0001)$ increase in the wall. Corresponding enlargements in myocyte cell volume per nucleus with failure were $71 \%(P$ $<0.0001), 37 \%(P<0.0001)$, and $53 \%(P<0.0001)$. This disproportionate expansion in volume of endomyocardial myocytes resulted in a difference in size between endocardial and epicardial myocytes. In failing animals, endocardial myocytes were 16\% $(P<0.001)$ larger than epicardial myocytes. Moreover, the hypertrophic response of endocardial myocytes in failing ventricles was $24 \%(P<0.001)$ greater than that of endocardial myocytes in ventricles with myocardial dysfunction. Finally, average myocyte cell volume per nucleus in the wall was $14 \%(P<0.01)$ larger in animals with failure than in those with ventricular dysfunction.

In summary, chronic coronary artery constriction led to myocyte cellular hypertrophy which was greater in the presence of ventricular failure than myocardial dysfunction.

Correlation analysis. Table II illustrates the results of regression analysis correlating various functional and anatomical parameters with respect to the reduction in cross-sectional area of the coronary artery lumen. This type of statistical evaluation was performed separately in animals with left ventricular dysfunction (group 1), left ventricular failure (group 2), and in the entire experimental animal population (group $1+$ group 2). Measurements of ventricular performance, including LVEDP, LVPSP, $+\mathrm{d} P / \mathrm{d} t$, and $-\mathrm{d} P / \mathrm{d} t$, did not correlate with the magnitude of coronary artery narrowing. Similarly, the typical aspects of ventricular remodeling, represented by chamber diameter, wall thickness, myocyte cell volume per nucleus, and total number of myocyte nuclei, were not correlated with the degree of coronary artery stenosis.

\section{Discussion}

The findings of the current study demonstrate that chronic constriction of the left main coronary artery of one month duration was associated with alterations in cardiac performance consisting of left ventricular dysfunction and failure. These two abnormal hemodynamic states were found in combinations with extensive ventricular remodeling characterized by chamber enlargement and thinning of the wall. These anatomical variations in conjunction with the depressed function provoked a marked elevation in diastolic ventricular stress. Moreover, discrete areas of myocardial injury characterized by sites of acute myocytolytic necrosis and multiple foci of replacement fibrosis in various phases of healing were found. The magnitude of myocyte loss was modest, accounting for $10 \%$ and $20 \%$ of the total myocyte population in the presence of ventricular dysfunction and failure, respectively. The myocyte cellular hypertrophic response exceeded the extent of cells being lost since the corresponding increases in myocyte cell volume per nucleus were $34 \%$ and $53 \%$. Therefore, a fixed lesion of a major epicardial artery led to severe impairment of left side pump dynamics in spite of reactive growth mechanisms in 

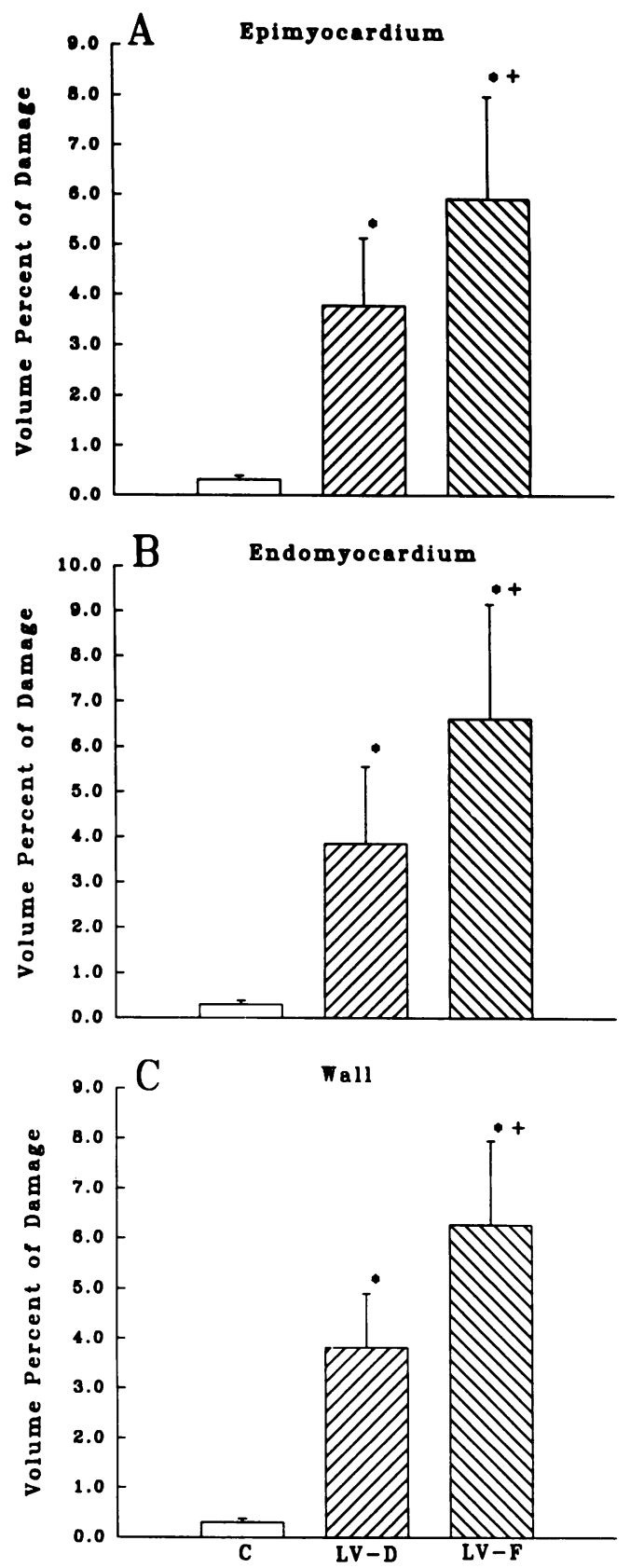

Figure 6. Effects of chronic coronary artery narrowing on the volume percent of myocardial damage in the left ventricular wall. Results are presented as mean \pm SD. *Value significantly different from the corresponding result in control animals (C). ${ }^{+}$Value significantly different from the corresponding result in animals with left ventricular dysfunction. (C, $n=8$; LV-D, $n=7$; LV-F, $n=10$.)

myocytes which resulted in an increase in muscle mass of the affected ventricle.

Myocyte loss and ventricular performance. Data in this investigation indicate that obstructions of the left coronary artery near its origin, generating a $45 \%$ average reduction in vessel luminal diameter, were accompanied by either a $10 \%$ loss of cells and ventricular dysfunction, or a $20 \%$ cell loss and ventricular failure. Thus, important differences exist between the effects of chronic coronary narrowing and those of total coronary occlusion on cardiac hemodynamics. Alterations in ventricular pump performance have been reported in rats with healed myocardial infarcts comprising $40 \%$ or more of the cells of the left ventricular wall $(17-19,21)$. With such large infarcts, left ventricular failure with elevated filling pressure, reduced cardiac output and a minimal capacity to respond to preload and afterload stresses has been encountered (17). However, with relatively small infarcts, comprising up to $35 \%$ of the left ventricle, no detectable impairment of cardiac function has been found $(17,21)$.

A similar discrepancy between the impact of coronary narrowing and occlusion on ventricular function has been dem-
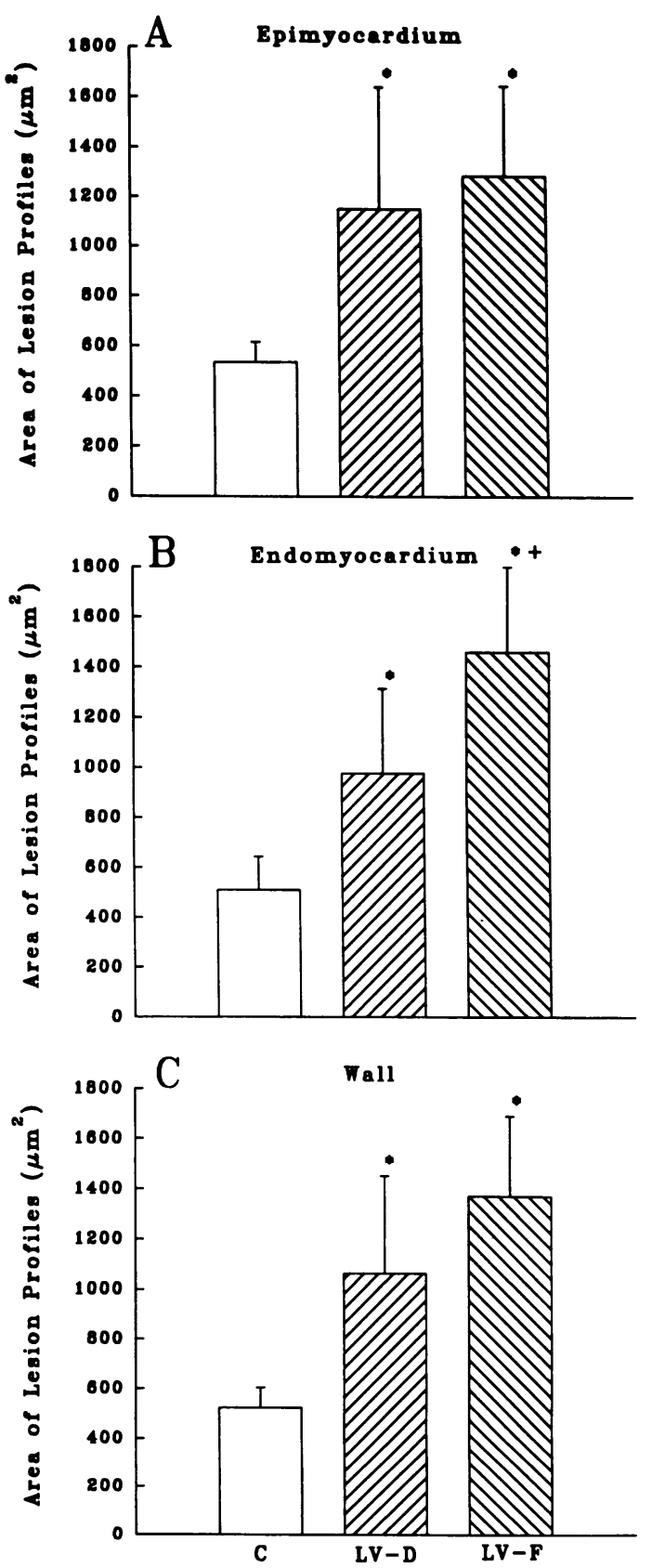

Figure 7. Effects of chronic coronary artery narrowing on the size of the lesion profiles in the left ventricular wall. Results are presented as mean \pm SD. *Value significantly different from the corresponding result in control animals $(\mathrm{C}) .{ }^{+}$Value significantly different from the corresponding result in animals with left ventricular dysfunction. (C, $n=8 ;$ LV-D, $n=7$; LV-F, $n=10$.) 

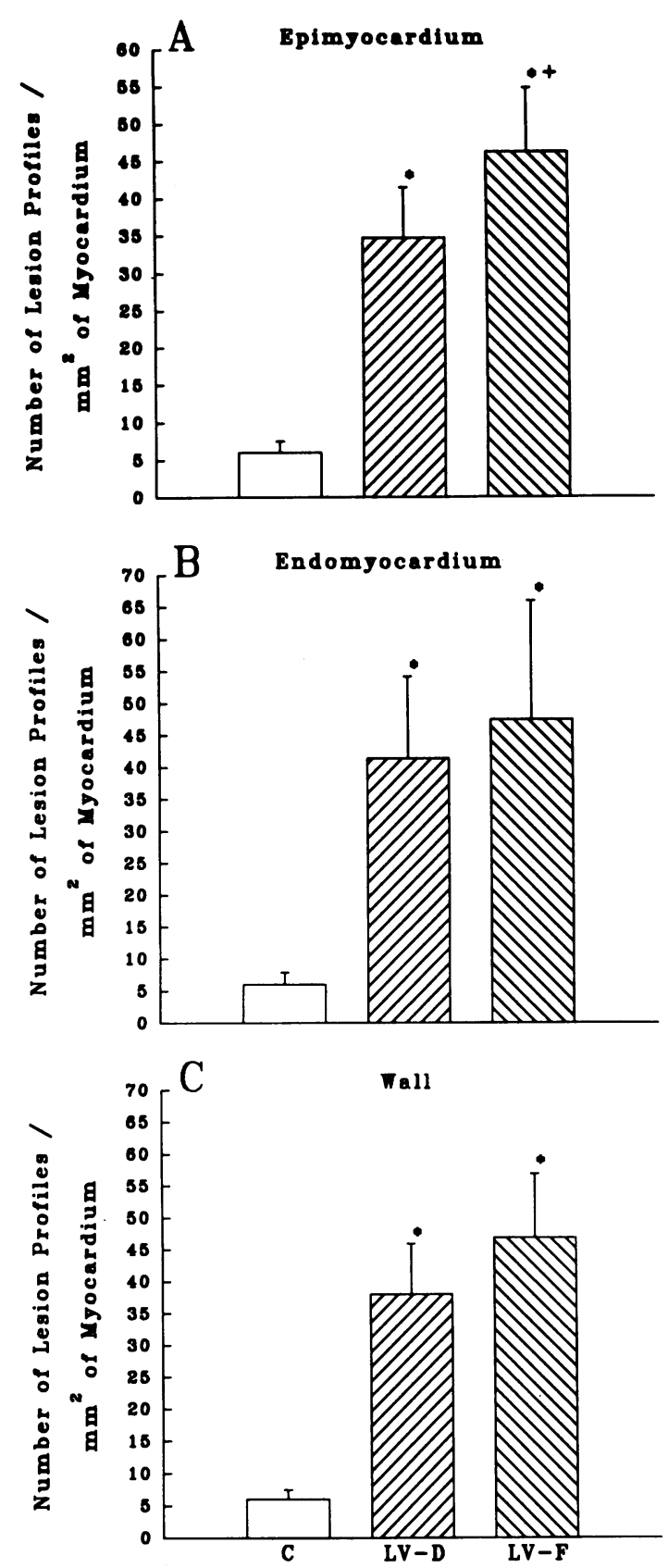

Figure 8. Effects of chronic coronary artery narrowing on the number of lesion profiles in the left ventricular wall. Results are presented as mean \pm SD. *Value significantly different from the corresponding result in control animals (C). ${ }^{+}$Value significantly different from the corresponding result in animals with left ventricular dysfunction. $(C$, $n=8 ;$ LV-D, $n=7$; LV-F, $n=10$.)

onstrated acutely after the reduction in caliber of the vessel lumen. Whereas with complete obstruction of a major epicardial artery and myocardial infarction, large destructions in muscle mass are required to impair myocardial contractile performance $(15,16)$, coronary stenosis and relatively moderate myocardial damage are accompanied by an immediate (24) and persistent (25) depression in left side pump dynamics. On the basis of these observations, the possibility may be advanced that a sudden change in coronary artery diameter may result in scattered loss of myocytes and profound impairment in ventric-

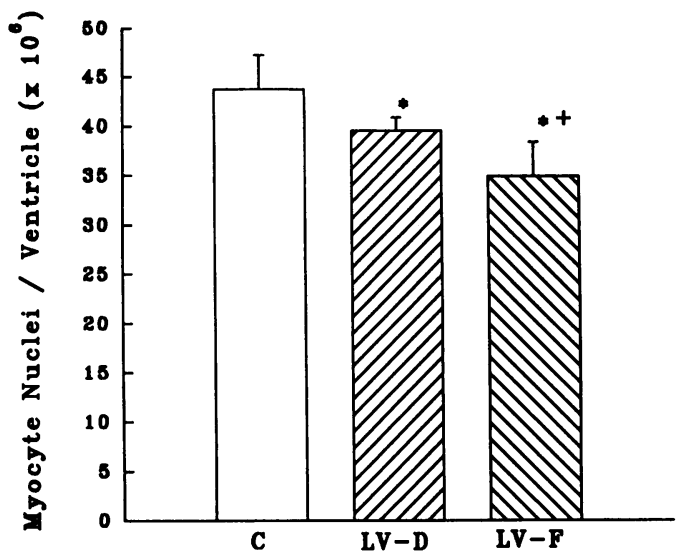

Figure 9. Effects of chronic coronary artery narrowing on the total number of myocyte nuclei in the left ventricle. Results are presented as mean $\pm \mathrm{SD}$. *Value significantly different from the corresponding result in control animals (C). ${ }^{+}$Value significantly different from the corresponding result in animals with left ventricular dysfunction. (C, $n=9$; LV-D, $n=7$; LV-F, $n=10$.)

ular performance $(24,33)$ which may reverse if the abnormality in the vessel wall resolves (33). On the other hand, a fixed lesion of the coronary artery, modest in nature, may provoke chronic and diffuse myocyte death (25) which may constitute a form of myocardial damage capable of continuously offsetting the reserve compensatory mechanisms of the injured ventricle, leading to irreversible myocardial dysfunction and overt congestive heart failure. Caution should be exercised in the translation of these experimental studies to the human population affected by atherosclerosis of the coronary arteries. Atherosclerosis is a disease state that progresses over a number of years, although acute hemorrhage within the atherosclerotic plaque may generate sudden constrictions in vessel luminal diameter.

Finally, it should be recognized that the evaluation of myocyte cell loss based on the changes in the number of myocyte nuclei in the ventricle may be influenced by a change in the proportion of mononucleated cells to multinucleated cells. However, the number of nuclei per myocyte has been found to be essentially unchanged in left ventricular dysfunction and failure $(27,32)$. On the other hand, it cannot be excluded that the calculated percent loss of myocytes following coronary artery stenosis may have been partially affected by such a phenomenon.

Characteristics of myocyte loss and ventricular size. The quantitative estimates of the magnitude of myocyte loss associated with coronary artery narrowing discussed above do not provide information on the distribution of this phenomenon on the ventricle. However, the morphometric analysis of the volume percentage, number, and size of lesion foci in the tissue demonstrated that damage to the myocardium involved the entire ventricular wall but was more severe in the endomyocardium. Moreover, the magnitude of this form of damage was greater in animals with left ventricular failure than in those with cardiac dysfunction. Importantly, myocytolytic necrosis with acute loss of discrete groups of cells was present one month after the surgical imposition of coronary stenosis. Finally, these pathological structural changes were found in combination with a marked increase in ventricular cavitary volume. 

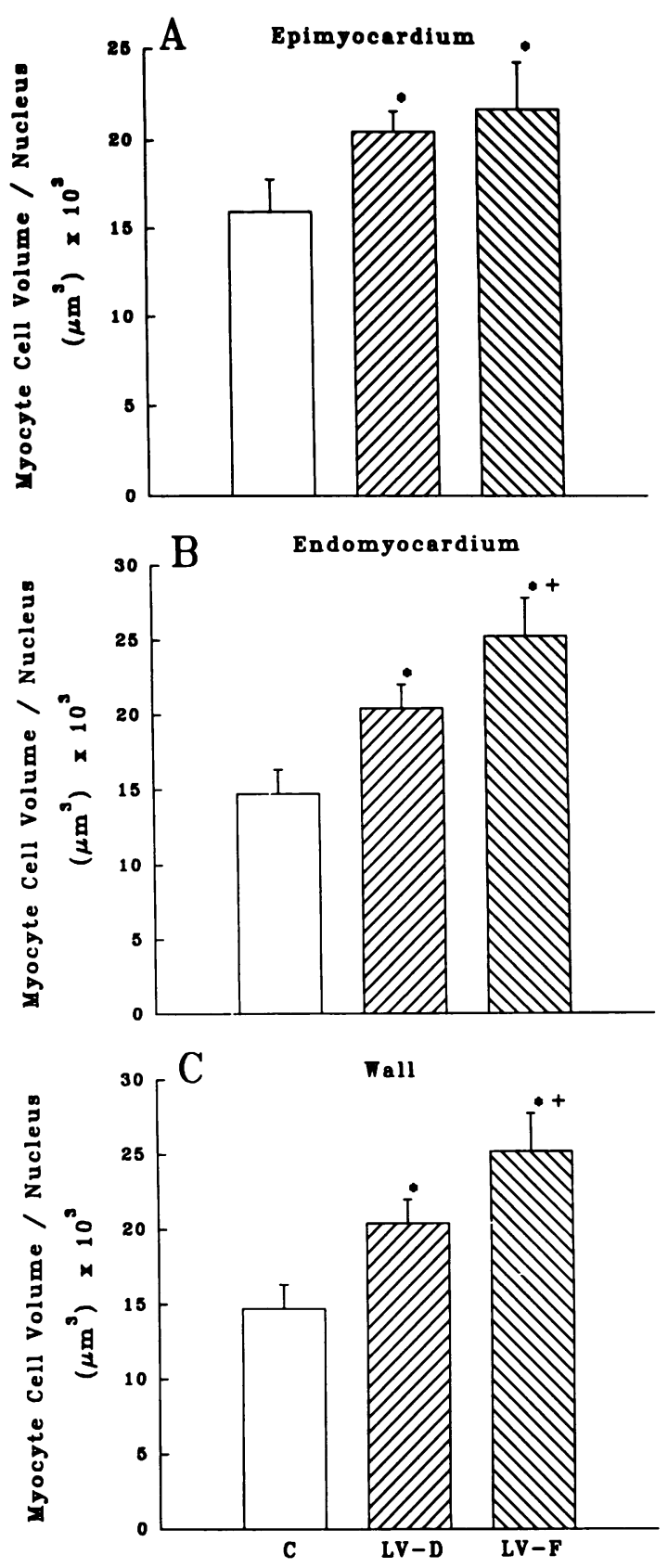

Figure 10. Effects of chronic coronary artery narrowing on myocyte size in the left ventricle. Results are presented as mean \pm SD. *Value significantly different from the corresponding result in control animals (C). ${ }^{+}$Value significantly different from the corresponding result in animals with left ventricular dysfunction. (C, $n=9$; LV-D, $n=7$; LV-F, $n=10$.)

The possibility that ongoing isolated myocytolytic necrosis plays a central role in the genesis of chamber enlargement is supported here by the extent of ventricular dilatation observed following coronary constriction. In contrast, a single episode of extensive myocyte necrosis in a segmental fashion $(15,21)$ does not provoke an expansion in cavitary volume unless regional damage involves $<35 \%$ of the entire ventricular wall, early (16) and late (19-21) after the ischemic event. Moreover, similar observations have been made in humans (34-36). Then, the question is whether focal myocardial damage is sufficient to explain the changes in ventricular cavitary volume or whether additional mechanisms have to be considered to account for the augmentation in ventricular size.

Cell loss, discrete in nature, can affect ventricular dimensions in two ways: acutely and chronically. The acute consequences reflect the necrotic phase in which dying myocytes are overstretched in diastole and noncontracting in systole, leading to diastolic and systolic wall thinning. On the other hand, the chronic effects include the reparative processes associated with healing and scar formation. On this basis, the increase in diastolic cavitary diameter should be identical to the decrease in wall thickness produced by the fraction of damage in the wall. Although this calculation exaggerates this phenomenon because it assumes that tissue injury does not contribute to wall thickness and myocytes do not hypertrophy laterally, augmentations in chamber diameter of $3 \%$ and $6 \%$ had to be expected with dysfunction and failure. However, cavitary diameter, halfway between the base and the apex, increased respectively by $23 \%$ and $32 \%$. Moreover, consistent with previous results (24) only small variations in sarcomere length occurred in this animal model in spite of an increase in end-diastolic pressure. In this regard, a decrease in the passive extensibility of sarcomeres has been observed in chronic left ventricular dilation (37), established cardiac hypertrophy (38), and acute left ventricular failure (39) in different model systems. Thus, thinning of the wall and lengthening of sarcomeres in myocytes can account only in part for the magnitudes of ventricular dilation measured here one month after coronary narrowing.

The mechanism responsible for ventricular wall and chamber remodeling after nonocclusive constrictions of major coronary arteries is at present unknown. However, the possibility may be raised that architectural rearrangement of myocytes with side-to-side slippage of muscle cells may have contributed to the expansion in cavitary volume (24). Side-to-side slippage of myocytes within the wall (16) would generate a reduction in the mural number of cells, wall thinning and chamber enlargement in the transverse direction. The lateral sliding of myocytes from the endocardial to the epicardial surface of the ventricular wall can also be anticipated to result in an increase in the longitudinal axis of the heart proportional to the extent of cell slippage. Thus, the decrease in the number of cells in the transverse orientation leads to an increase in the number of cells in the longitudinal axis. The major axis of the ventricle will then increase by a factor involving number of cells, average cell diameter and the angle of orientation of muscle cells with respect to the longitudinal diameter of the heart.

The observations here of increases in the longitudinal and transverse diameters with thinning of the wall in spite of a cellular hypertrophic response suggest that acute and chronic mechanisms have both been operative during the one month interval studied. The expansion in cavitary volume may be the critical determinant in altering the capacity of the heart to restore normal cardiac performance. Should the mechanism of cell movement be effective in the presence of coronary narrowing, acute and chronic myocytolytic necrosis would have to be regarded as an important, but secondary event dictated by the mechanical consequences generated by the interaction of wall remodeling and ventricular pressure $(24,25)$. Moreover, the overall expansion in ventricular cavitary volume creates a mechanical disadvantage to the myocytes in the wall by increasing diastolic mural stress when fewer cells are present to handle the load. As a result of these anatomical and functional adaptations, a hypertrophic reaction of myocytes is elicited and the 
Table II. Correlation Analysis between Coronary Artery Constriction and Physiologic and Anatomic Parameters

\begin{tabular}{|c|c|c|c|c|c|c|c|c|c|}
\hline Group & $\%$ Area & LVEDP & LVPSP & $+\mathrm{d} P / \mathrm{d} t$ & $-\mathrm{d} P / \mathrm{d} t$ & $\mathrm{CD}$ & WT & CS & $\mathrm{CN}$ \\
\hline \multirow{2}{*}{$1(n=8)$} & $r=$ & 0.510 & 0.180 & -0.440 & -0.490 & -0.150 & 0.370 & 0.450 & 0.120 \\
\hline & $P=$ & 0.190 & 0.660 & 0.280 & 0.210 & 0.720 & 0.630 & 0.310 & 0.780 \\
\hline \multirow{2}{*}{$2(n=11)$} & $r=$ & -0.030 & -0.230 & -0.080 & -0.220 & 0.350 & -0.240 & -0.040 & -0.030 \\
\hline & $P=$ & 0.920 & 0.510 & 0.810 & 0.520 & 0.330 & 0.500 & 0.920 & 0.940 \\
\hline \multirow{2}{*}{$1+2(n=19)$} & $r=$ & 0.004 & -0.041 & 0.025 & -0.054 & 0.058 & 0.066 & -0.046 & 0.136 \\
\hline & $P=$ & 0.990 & 0.860 & 0.910 & 0.820 & 0.810 & 0.800 & 0.850 & 0.610 \\
\hline
\end{tabular}

Linear regression analysis comparing the relation between the degree of coronary artery constriction, as a percentage of the total luminal crosssectional area and various parameters of cardiac function and structure. Abbreviations: $+\mathrm{d} P / \mathrm{d} t$, peak rate of left ventricular pressure rise; $-\mathrm{d} P / \mathrm{d} t$, peak rate of left ventricular pressure decay; $\mathrm{CD}$, left ventricular chamber diameter; WT, left ventricular wall thickness; CS, myocyte cell size per nucleus, $\mathrm{CN}$, total number of myocyte nuclei; $r$, correlation coefficient; $P, P$ value.

nature of the mechanical stimulus will determine a prevailing increase in cell length with additional chamber dilation. Data consistent with this sequence of events have been obtained after acute (16) and chronic (21) myocardial infarction.

Myocyte hypertrophy and wall stress. Findings in this investigation indicate that myocyte hypertrophy occurred as a result of coronary artery narrowing and the magnitude of cellular enlargement was greater in animals with left ventricular failure, $53 \%$, than in rats with myocardial dysfunction, $34 \%$. In spite of these cellular growth mechanisms, which not only compensated but exceeded the amount of muscle mass lost by focal myocyte cell death, diastolic wall stress was markedly elevated. Since myocardial hypertrophy was $23 \%$ and $30 \%$ in the two groups of animals whereas intracavitary volume increased $63 \%$ and $93 \%$, the ventricular mass-to-chamber volume ratio decreased $25 \%$ and $33 \%$ with dysfunction and failure.

It is the current view that myocyte growth in hypertrophy is proportional to the amount of stress imposed on the heart (40, 41). Increasing pressure load on the adult heart induces concentric ventricular hypertrophy in which wall thickness increases without chamber enlargement (40). This phenomenon is accomplished by lateral expansion of myocytes with no change in the number of cells across the wall or in average myocyte length (42). According to the law of Laplace, the greater myocyte diameter would produce a proportional thickening of the wall that should offset the higher peak systolic wall stress resulting from the elevation in pressure $(40,41)$. In contrast, an increasing volume load induces enlargement of the ventricular chamber without a relative increase in its wall thickness, that is, compensated eccentric hypertrophy (42). Chamber dilatation is accompanied by a proportional increase in wall thickness so that the ratio of wall thickness-to-chamber radius remains constant (42). At the cellular level, chamber enlargement appears to be brought about through lengthening of myocytes with only slight variations in myocyte cross sectional area and no change in sarcomere length (42). Lengthening of myocytes would have the effect of counteracting the greater end-diastolic wall stress $(40,41)$ by contributing to the enlargement in chamber volume and the decrease in diastolic filling pressure. However, when the relationship between chamber size and wall thickness is not maintained, decompensated eccentric hypertrophy develops (43), as appears to be the case in chronic coronary artery constriction in which wall thickness-to-chambers radius ratio decreased from base to apex in all animals. Consistent with previous observations $(24,25)$, diastolic overload may represent the prevailing mechanical stimulus implicated in the acute (24), subacute (25), and chronic growth responses of the myocardium. This chronic effect has been documented here 1 mo after surgery.

Although the analysis of the functional and anatomical characteristics of the left ventricle after coronary artery narrowing allowed the identification of the overload responsible for the hypertrophic adaptation of the unaffected myocardium, more difficult is the understanding of the hypertrophic reaction of the right ventricle in the presence of normal and abnormal right side function. Right ventricular hypertrophy was accompanied by an increase in average wall thickness which was comparable to the expansion in right ventricular weight in animals with preserved right ventricular dynamics but significantly less in rats with impaired right ventricular performance. Such a phenomenon implies an increase in right ventricular chamber volume (42) in the latter group which, in combination with the altered function, suggests that a diastolic Laplace overload was operative on the right ventricle as well.

Temporal effects of coronary artery narrowing. The results in the current study significantly extend previous observations obtained in our laboratory in this animal model $(24,25)$. Although myocardial damage and impairment in cardiac function were previously documented, questions remained whether hypertrophy of the unaffected cells was capable of restoring cardiac pump performance and whether myocyte damage persisted over time creating a chronic ongoing process. Findings in this investigation clearly indicate that myocyte hypertrophy does not normalize myocardial dysfunction, and that cell loss continues to occur at one month after coronary artery narrowing. Importantly, the magnitude of myocyte loss was modest but sufficient to engender severe ventricular dilation and contribute to the maintenance of depressed contractility. Finally, the interaction between the abnormality in myocardial structure and impaired ventricular dynamics was found to ensue a sustained elevation in ventricular loading.

In conclusion, a fixed reduction in luminal diameter of the left main coronary artery leads to chronic and diffuse myocyte loss with extensive ventricular remodeling, decompensated eccentric hypertrophy, and severe deterioration in ventricular pump performance which may progress into overt congestive heart failure and death, mimicking the cardiomyopathic heart in humans.

\section{Acknowledgments}

The invaluable technical assistance of Lucille Robinson is greatly appreciated. 
This work was supported by National Institutes of Health grants HL-38132, HL-39902, and HL-40561 and a grant-in-aid from the American Heart Association, New York State Affiliate, Inc.

\section{References}

1. Roberts, W. C., and A. A. Jones. 1979. Quantitation of coronary arterial narrowing at necropsy in sudden coronary death: analysis of 31 patients and comparison with 25 control subjects. Am. J. Cardiol. 44:39-45.

2. Oalmann, M. C., R. W. Palmer, M. A. Guzman, and R. A. Strong. 1980. Sudden death, coronary heart disease, atherosclerosis and myocardial lesions in young men. Am. J. Epidemiol. 112:639-649.

3. Roeske, W. R., R. M. Savage, R. A. O'Rourke, and C. M. Bloor. 1981 Clinicopathologic correlations in patients after myocardial infarction. Circulation. 63:36-45.

4. Warnes, C. A., and W. C. Roberts. 1984. Sudden coronary death: relation of amount and distribution of coronary narrowing at necropsy to previous symptoms of myocardial ischemia, left ventricular scarring and heart weight. Am. J. Cardiol. 54:65-73.

5. White, C. W., C. B. Wright, D. B. Doty, L. F. Hiratza, C. L. Eastham, D. G. Harrison, and M. L. Marcus. 1984. Does the visual interpretation of the coronary arteriogram predict physiological significance of a coronary stenosis. $\mathrm{N}$. Engl. J. Med. 310:819-824.

6. Harrison, D. G., C. W. White, L. F. Hiratzka, D. B. Doty, D. H. Barnes, C. L. Eastham, and M. L. Marcus. 1984. The value of lesion cross-sectional area determined by quantitative coronary angiography in assessing the physiologic significance of proximal left anterior descending coronary arterial stenoses. Circulation. 69:1111-1119.

7. Buja, M. L., and J. T. Willerson. 1987. The role of coronary artery lesions in ischemic heart disease: insights from recent clinicopathologic, coronary arteriography and experimental studies. Hum. Pathol. 18:451-461.

8. Roberts, W. C. 1976 . The coronary arteries and left ventricle in clinically isolated angina pectoris: a necropsy analysis. Circulation. 54:388-390.

9. Schuster, E. H., and B. H. Bulkley. 1980. Ischemic cardiomyopathy: a clinicopathologic study of fourteen patients. Am. Heart J. 100:506-512.

10. Roberts, C. S., and W. C. Roberts. 1980. Cross-sectional area of the proximal portions of the three major epicardial coronary arteries in 98 necropsy patients with different coronary events. Circulation. 62:953-959.

11. Buja, L. M., and J. T. Willerson. 1981. Clinicopathologic correlates of acute ischemic heart disease syndromes. Am. J. Cardiol. 47:343-356.

12. Pantely, G. A., and J. D. Bristow. 1984. Ischemic cardiomyopathy. Prog. Cardiovasc. Dis. 27:95-114.

13. Harnarayan, C., M. A. Bennet, B. L. Pentecost, and D. B. Brewer. 1970. Quantitative study of infarcted myocardium in cardiogenic shock. Br. Heart J. 32:728-732.

14. Page, D. L., J. B. Caulfield, J. A. Kastor, R. W. DeSanctis, and C. A. Sanders. 1971. Myocardial changes associated with cardiogenic shock. N. Engl. J. Med. 285:133-137.

15. Anversa, P., A. V. Loud, V. Levicky, and G. Guideri. 1985. Left ventricular failure induced by myocardial infarction. I. Myocyte hypertrophy. Am. J. Physiol. 248:H876-H882.

16. Olivetti, G., J. M. Capasso, E. H. Sonnenblick, and P. Anversa. 1990. Side-to-side slippage of myocytes participates in ventricular wall remodeling acutely after myocardial infarction in rats. Circ. Res. 67:23-34.

17. Pfeffer, M. A., J. M. Pfeffer, M. C. Fishbein, P. J. Fletcher, J. Spadaro R. A. Kloner, and E. Braunwald. 1979. Myocardial infarct size and ventricular function in rats. Circ. Res. 44:503-512.

18. Fletcher, P. J., J. M. Pfeffer, M. A. Pfeffer, and E. Brauwald. 1981. Left ventricular diastolic pressure-volume relations in rats with healed myocardial infarction. Circ. Res. 49:618-626.

19. Pfeffer, J. M., M. A. Pfeffer, and E. Braunwald. 1985. Influence of chronic captopril therapy on the infarcted left ventricle of the rat. Circ. Res. 57:84-95.

20. Pfeffer, M. A., J. M. Pfeffer, C. Steinberg, and P. Finn. 1985. Survival after an experimental myocardial infarction: beneficial effects of long-term therapy with captopril. Circulation. 72:406-412.
21. Olivetti, G., J. M. Capasso, L. G. Meggs, E. H. Sonnenblick, and P. Anversa. 1991. Cellular basis of chronic ventricular remodeling after myocardial infarction in rats. Circ. Res. 68:856-869.

22. Feild, B. J., R. O. Russell, J. T. Dowling, and C. E. Rackley. 1972. Regional left ventricular performance in the year following myocardial infarction. Circulation. 46:679-689.

23. Taylor, G. J., J. O. Humphries, E. D. Mellitis, B. Pitt, R. A. Schulze L. S. C. Griffith, and S. C. Achoff. 1980. Predictors of clinical course, coronary anatomy and left ventricular function after recovery from acute myocardial infarction. Circulation. 62:960-970.

24. Capasso, J. M., M. J. Jeanty, T. Palackal, G. Olivetti, and P. Anversa. 1989. Ventricular remodeling induced by acute nonocclusive constriction of coronary artery in rats. Am. J. Physiol. 257:H1983-H1993.

25. Capasso, J. M., P. Li, and P. Anversa. 1991. Nonischemic myocardial damage induced by nonocclusive constriction of coronary artery in rats. $\mathrm{Am}$. $\mathrm{J}$. Physiol. 260:H651-H661.

26. Johns, T. N. P., and B. J. Olson. 1954. Experimental myocardial infarction. I. A method of coronary occlusion in small animals. Ann. Surg. 140:675682

27. Capasso, J. M., T. Palackal, G. Olivetti, and P. Anversa. 1990. Severe myocardial dysfunction induced by ventricular remodeling in aging rat hearts. Am. J. Physiol. 259:H1086-H1096.

28. Capasso, J. M. T. Palackal, G. Olivetti, and P. Anversa. 1990. Left ventricular failure induced by long-term hypertension in rats. Circ. Res. 66:1400-1412.

29. Anversa, P., C. Beghi, Y. Kikkawa, and G. Olivetti. 1986. Myocardial infarction in rats: Infarct size, myocyte hypertrophy and capillary growth. Circ. Res. 58:26-37.

30. Loud, A. V., P. Anversa. 1984. Morphometric analysis of biologic processes. Lab. Invest. 50:250-261.

31. Wallenstein, S., C. L. Zucker, and J. L. Fleiss. 1980. Some statistical methods useful in circulation research. Circ. Res. 47:1-9.

32. Anversa, P., T. Palackal, E. H. Sonnenblick, G. Olivetti, L. G. Meggs, and J. M. Capasso. 1990. Myocyte cell loss and myocyte cellular hyperplasia in the hypertrophied aging rat heart. Circ. Res. 67:871-885.

33. Maseri, A., and S. Chierchia. 1982. Coronary artery spasm: demonstration, definition, diagnosis and consequences. Prog. Cardiovasc. Dis. 25:169-192.

34. McKay, R. G., M. A. Pfeffer, R. C. Pasternak, J. E. Markis, G. C. Come, C. Nakao, J. D. Alderman, J. J. Ferguson, R. D. Safian, and W. Grossman. 1986. Left ventricular remodeling after myocardial infarction: a corollary to infarct expansion. Circulation. 74:693-702.

35. Warren, S. E., H. D. Royal, J. E. Markis, W. Grossman, and R. J. McKay. 1988. Time course of left ventricular dilatation after myocardial infarction: influence of infarct-related artery and success of coronary thrombolysis. J. Am. Coll. Cardiol. 11:12-19.

36. Pfeffer, M. A., G. A. Lamas, D. E. Vaughan, A. F. Parisi, and E. Braunwald. 1988. Effect of captopril on progressive ventricular dilatation after anterior myocardial infarction. N. Engl. J. Med. 319:80-86.

37. Ross, J., Jr., E. H. Sonnenblick, R. R. Taylor, H. M. Spotniz, and J. W. Covell. 1971. Diastolic geometry and sarcomere lengths in the chronically dilated canine left ventricle. Circ. Res. 28:49-61.

38. Anversa, P., L. Vitali-Mazza, O. Visioli, and G. Marchetti. 1971. Experimental cardiac hypertrophy: a quantitative ultrastructural study in the compensatory stage. J. Mol. Cell. Cardiol. 3:213-227.

39. Vitali-Mazza, L., P. Anversa, F. Tedeschi, R. Mastandrea, V. Mavilla, and O. Visioli. 1972. Ultrastructural basis of acute left ventricular failure from severe acute aortic stenosis in the rabbit. J. Mol. Cell. Cardiol. 4:661-671.

40. Grossman, W., D. Jones, and L. P. McLaurin. 1975. Wall stress and patterns of hypertrophy in the human left ventricle. J. Clin. Invest. 56:56-64.

41. Grossman, W., B. A. Carabello, S. Gunther, and M. A. Fifer. 1983. Ventricular wall stress and the development of cardiac hypertrophy and failure. In Myocardial Hypertrophy and Failure. N. R. Alpert, editor. Perspect. Cardiovasc. Res. 7:1-18.

42. Anversa, P., R. Ricci, and G. Olivetti. 1986. Quantitative structural analysis of the myocardium during physiologic growth and induced cardiac hypertrophy: a review. J. Am. Coll. Cardiol. 7:1140-1149.

43. Linzbach, A. J. 1960. Heart failure from the point of view of quantitative anatomy. Am. J. Cardiol. 5:370-382. 